\title{
Community-acquired methicillin-resistant Staphylococcus aureus: the role of Panton-Valentine leukocidin
}

\author{
Susan Boyle-Vavra and Robert S Daum \\ Department of Pediatrics, Section of Infectious Diseases, University of Chicago, Chicago, IL, USA
}

\begin{abstract}
Community-acquired (CA) methicillin-resistant Staphylococcus aureus (MRSA) infection among individuals without healthcare-associated (HA) risk factors was first recognized about a decade ago. It has now emerged as an epidemic that is responsible for rapidly progressive, fatal diseases including necrotizing pneumonia, severe sepsis and necrotizing fasciitis. Unlike HA-MRSA, CA-MRSA are usually pan-susceptible to non- $\beta$-lactam antimicrobials. In addition to novel methicillin resistance genetic cassettes, many CA-MRSA harbor a phage harboring Panton-Valentine Leukocidin (PVL) genes and some data support the idea that PVL is responsible at least in part for the increased virulence of CA-MRSA. The tight association between the novel methicillin resistance cassettes and PVL phage cannot be explained, as they integrate into distinct sites on the $S$. aureus chromosome. This paper presents the evidence that CA-MRSA isolates are distinct strains emerging de novo from CA-methicillin susceptible isolates rather than from HA-MRSA isolates that have escaped from the hospital setting and that these novel CA-MRSA isolates may be more virulent than HA-MRSA. The second aim is to outline the progress in understanding the role of PVL in CA-MRSA pathogenesis.
\end{abstract}

Laboratory Investigation (2007) 87, 3-9. doi:10.1038/labinvest.3700501; published online 4 December 2006

Keywords: panton-valentine leukocidin virulent; methicillin resistance; community-acquired; staphylococcus aureus; necrotizing pneumonia; severe sepsis

Staphylococcus aureus, the most virulent Staphylococcus species, is also the most prevalent pathogen isolated from hospitalized patients and the second most common from patients in outpatient settings. $S$. aureus causes a wide range of syndromes, from minor skin and soft tissue infection to life-threatening pneumonia and toxinoses such as toxic shock syndrome. ${ }^{1}$ The high mortality due to $S$. aureus was abated by penicillin in the 1940s, but this was short lived as penicillin-resistant $S$. aureus (PRSA) producing $\beta$-lactamase quickly emerged and $90 \%$ of hospital-acquired $S$. aureus were penicillin-resistant within 10 years.

Methicillin, a $\beta$-lactamase-insensitive $\beta$-lactam, provided new treatment options for PRSA infections in the late 1950s, but methicillin-resistant $S$. aureus (MRSA) that are crossresistant to all $\beta$-lactams, including penicillins and cephalosporins, soon emerged, primarily in healthcare environments.

Correspondence: Dr S Boyle-Vavra, PhD, Department of Pediatrics, Section of Infectious Diseases, University of Chicago MC6054, Chicago, IL 60637, USA.

E-mail: sboyle@peds.bsd.uchicago.edu

Received 26 September 2006; revised and accepted 12 October 2006; published online 4 December 2006
MRSA isolates became multiply resistant to other classes of antimicrobials, pushing the 'antibiotic of last resort', vancomycin, into exponentially increasing use. Rates of methicillin resistance increased slowly, but progressively, until the late 1990s when a dramatic surge in MRSA rates began. ${ }^{2}$ This increase was accompanied by the isolation of MRSA isolates from community-acquired (CA) infections among previously healthy individuals with few or no traditional healthcare-associated (HA) risk factors for MRSA. ${ }^{3}$ This represented a dramatic change in the epidemiology of MRSA since CA-S. aureus infection had been previously caused mainly by methicillin-susceptible $S$. aureus (MSSA) strains.

Since these early reports, CA-MRSA isolates have become globally pervasive; ${ }^{4}$ and reports of serious and rapidly progressive fatal disease due to virulent CA-MRSA have alarmed healthcare professionals and the lay media alike. ${ }^{5,6}$ In many locations CA-MRSA isolates have become more prevalent than MSSA. ${ }^{7}$

Why CA-MRSA is an Important Clinical Problem?

This CA-MRSA epidemic has made $\beta$-lactams, which were once uniformly effective against CA-S. 
4

\section{The Panton-Valentine Leukocidin Associated with CA-MRSA}

Epidemiologic and clinical data ${ }^{4,9}$ provide compelling evidence that the high virulence potential of CA-MRSA is associated with the genes $l u k S-P V$ and lukF-PV ( $p v l)$ encoding the subunits of the PantonValentine leukocidin (PVL). CA-MRSA isolates associated with disease bear pvl with nearly universal prevalence. PVL is a bicomponent, poreforming leukotoxin initially designated 'substance leukocidine' by Van deVelde in 1894 due to its ability to lyse leukocytes. Panton and Valentine first associated the leukotoxin with skin and soft tissue infection in 1932, long before PRSA or MRSA were of clinical concern. Subsequent identification of the $P V L$ genes solidified the association of $p v l$ with severe skin and soft tissue infection and necrotizing pneumonia among CA-MSSA isolates ${ }^{9,10}$ and subsequently among CA-MRSA isolates. ${ }^{4}$ Moreover, the clinical sequelae of $p v l$-positive infections tend to be more severe than pvl-negative $S$. aureus. ${ }^{9}$ For example, pneumonia associated with pvl-positive $S$. aureus is more frequently associated with sepsis, high fever, leukopenia, hemoptysis, pleural effusion and death. ${ }^{9}$ Similar to CA-MSSA, $p v l$ has been associated with epidemic CA-MRSA strains causing skin and soft tissue infections, necrotizing pneumonia and lethal necrotizing fasciitis. ${ }^{4-8}$ Moreover, despite the large repertoire of transferable toxin genes among $S$. aureus strains, including enterotoxigenic superantigens, exfoliative toxins and poreforming toxins homologous to PVL, ${ }^{11} \mathrm{pvl}$ is the most consistently present transferable toxin locus among CA-MRSA strains. ${ }^{4,8,12}$ Conversely, $p v l$ is less often present in CA-MRSA isolates associated with asymptomatic nasal colonization. ${ }^{13}$

\section{The Unexpected Origins of CA-MRSA}

CA-MRSA strains were initially thought to be nosocomial strains that had spread from hospital to community settings. However, the paradoxical susceptibility of CA-MRSA to non- $\beta$-lactam antimicrobials and the associated clinical syndromes more typical of MSSA strongly suggested that CA-MRSA strains were distinct from those prevalent in healthcare settings. ${ }^{3}$ The further discovery of genotypic differences that distinguish CA-MRSA from HA-MRSA has led to general acceptance of the view that CA-MRSA evolved from MSSA strains endemic in the community.

Three major genotypic markers distinguish CAMRSA strains from HA-MRSA isolates: their genetic lineage, the architecture of the methicillin resistance genetic elements and the presence of $p v l$.

Methicillin resistance is mediated by an acquired penicillin binding protein, PBP2a, a peptidoglycan transpeptidase encoded by the mecA gene that has low affinity for $\beta$-lactams. Thus, when the four native peptidoglycan synthetases (penicillin-binding proteins 1, 2, 3 and 4) are bound and inactivated by $\beta$-lactams, PBP2a can still effect cell-wall synthesis. mecA is harbored on the staphylococcal chromosomal cassette mec (SCCmec), a genetic element that integrates site specifically into the $S$. aureus chromosome. Horizontal transfer of SCCmec is implied ${ }^{14}$ although the means of transfer remains to be elucidated.

HA-MRSA usually carry large SCCmec types I, II or III $(34-67 \mathrm{~kb}),{ }^{15}$ but CA-MRSA isolates harbor newly described smaller SCCmec variants: either type IV ( $24 \mathrm{~kb}),{ }^{16,17}$ or less frequently, SCCmec V or a variant, $V_{\mathrm{T}} \cdot{ }^{13,14}$ The greater size of SCCmec II and III largely represents inclusion of non- $\beta$-lactam antibiotic resistance genes, which are usually lacking in SCCmec IV and V. Another important genetic difference between CA- and HA-MRSA is the presence of an integrated bacteriophage (phiSLT), carrying the $p v l$ genes ${ }^{4,18}$ in CA-MRSA. Pvl is widely associated with the presence of SCCmec IV and sporadically with SCCmec $V_{\mathrm{T}}$ or V but not SCCmec types I, II or III. SCCmec IV was found in commensal Staphylococcus epidermidis in the 1970s, before its first documentation in $S$. aureus in the $1980 \mathrm{~s},{ }^{19}$ suggesting that that SCCmec IV was transferred from S. epidermidis sometime in the 1980s.

Multilocus sequence typing (MLST) ${ }^{20}$ and pulsotyping using pulsed-field gel electrophoresis ${ }^{21}$ have been used to demonstrate that the genetic backgrounds of CA-MRSA isolates are distinct from predominant HA-MRSA clones circulating within defined geographic regions. Remarkably, the genetic backgrounds of CA-MRSA isolates are distinct in different geographic locations $s^{4,13,20}$ and, conversely, 
many different backgrounds can exist within a narrow geographic area. ${ }^{17}$ In the US, most CA-MRSA disease isolates initially carried SCCmec IV in the genetic backgrounds ST 1/pulsotype USA $400^{5}$ but the predominant CA-MRSA strain recently is ST 8/pulsotype USA $300 .^{7}$ These are distinct from the most prevalent multiresistant HA-MRSA clone in circulation in the US today, ST 5 that carries SCCmec II. ${ }^{12,21}$

The current accepted model to explain the origins of CA-MRSA (Figure 1) states that a small methicillin resistance cassette (eg, SCCmec IV) independently integrated into the genomes of many different MSSA ancestral clones circulating in different geographic regions. A few lineages endowed with the best fitness traits have survived. Indeed, the faster growth rates of CA-MRSA suggest that they are more fit than HA-MRSA. This model parallels the emergence and spread of HA-MRSA from MSSA in the $1960 \mathrm{~s}^{22}$ except that unlike HA-MRSA isolates, CA-MRSA isolates have likely emerged from MSSA carrying pvl. In some locations, the pvl locus is now more common among CA-MRSA isolates than CA-MSSA isolates ${ }^{7}$ suggesting that the methicillin resistance has contributed to the success of pvlpositive $S$. aureus strains.

Interestingly, the ST 8 lineage of the predominant CA-MRSA clone in the US is closely related to successful HA-MRSA and MSSA clones in Europe and the first MRSA strain isolated. ${ }^{22}$ This suggests that the ST 8 lineage easily accommodates insertion of an SCCmec element.

The recent worldwide emergence of different genetic backgrounds carrying both the phiSLT lysogenic phage carrying $p v l$ and one of the small methicillin resistance cassettes suggests a common, global selective pressure. Increased use of antimicrobials in the community cannot explain the presence of pvl. It is unlikely that phiSLT and small
SCCmec elements share the same vehicle for horizontal transfer since they integrate into distinct sites in the $S$. aureus chromosome.

The fact that PVL can cause dermonecrosis in rabbits $^{23}$ supports the idea that PVL itself might provide a selective advantage for dissemination of CA-MRSA among healthy individuals due to the production of open skin lesions in patients, from which bacteria can readily shed and infect close contacts. However, the frequent isolation from the nares of pvl-negative MRSA from asymptomatic healthy carriers suggests PVL is not required for dissemination by the respiratory route. ${ }^{13}$

A controversial, hypothesis to explain the increase in CA-MRSA is the decreased nasal colonization by competing Streptococcus pneumoniae due to the pneumococcal Prevnar ${ }^{\mathrm{TM}}$ vaccine. This hypothesis is supported by the observation that the rate of nasopharyngeal colonization by $S$. aureus is inversely proportional to that of pneumococcal serotypes targeted by the vaccine ${ }^{24}$ suggesting a competitive interaction in the nasopharynx whereby elimination of one pathogen opens a niche for others. However, would not explain the association of $p v l$ with CA-MRSA.

Increasingly, MRSA strains carrying $p v l$ and bearing CA-genotypes (SCCmec IV, ST 8) are being reported in HA infection. ${ }^{25}$ This may be due to preexisting colonization with pvl-positive CA-MRSA isolates that find a portal of entry during invasive procedures performed in the hospital. Alternately, CA-MRSA strains might now be endemic in certain hospitals.

\section{Evidence that PVL is a Major Virulence Factor in CA-MRSA}

Direct evidence that PVL plays a role in pathogenesis has been limited. While a role for PVL in

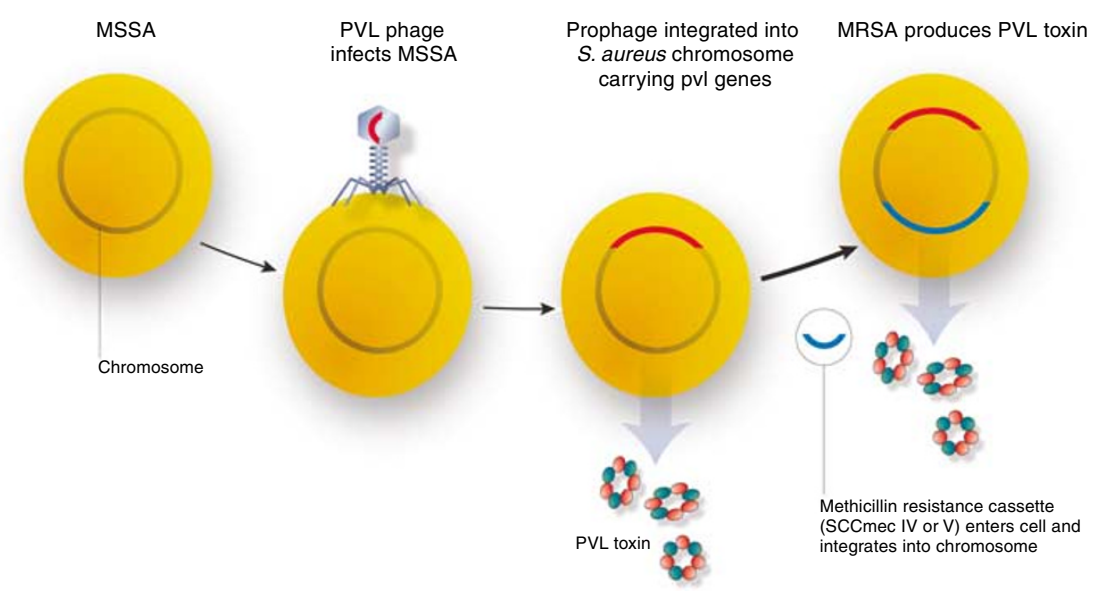

Figure 1 Model for emergence of PVL producing CA-MRSA: a methicillin-susceptible $S$. aureus (MSSA) strain is infected and lysogenized by a phage (phiSLT) that harbors the lukS-PV and lukF-PV genes ( $p v l$ ) encoding the PVL. Subsequently, a methicillin resistance cassette (SCCmec IV, $\mathrm{V}$ or $V_{\mathrm{T}}$ ) carrying the mecA gene is horizontally transferred into the pvl-positive MSSA strain and integrates into the genome in a location that is distinct from that of the phiSLT integration site. 
6

dermonecrosis in rabbits has been established, ${ }^{23,26,27}$ whether it also plays a role in necrotizing pneumonia, severe sepsis and necrotizing fasciitis is unknown.

The two components of PVL, LukS-PV and LukF$\mathrm{PV}$, are secreted before they assemble into a pore-forming heptamer on polymorphonuclear leukocytes (PMN) membranes ${ }^{11}$ (Figure 2), leading to PMN lysis. Unlike other $S$. aureus pore-forming leukocidins, PVL is not hemolytic. ${ }^{11}$ LukS-PV initiates binding to an unidentified receptor on PMNs where it subsequently dimerizes with LukFPV followed by alternate serial binding of LukF-PV and LukS-PV components until the heptamer is assembled. When LukS-PV binds PMNs, a host protein kinase (A or C) phosphorylates LukS-PV followed by induction of $\mathrm{Ca}^{++}$ion channels. ${ }^{11}$ This suggests induction of signal of transduction events that may trigger production of interleukins and inflammatory mediators. Depending on the concentration of PVL, it can cause either PMN lysis or apoptosis, the latter via a novel pathway that presumably involves PVL-mediated pore formation in the mitochondrial membrane ${ }^{28}$ (Figure 3).

Consistent with the PMN cytolytic activity of PVL, neutropenia is a frequent clinical finding associated with pvl-positive $S$. aureus necrotizing pneumonia. ${ }^{5,9}$ PVL-mediated PMN lysis and apoptosis ${ }^{28}$ may define the first step in pathogenesis by mediating evasion of this first line of host defense but the path leading to tissue necrosis and severe sepsis is unclear. Figure 2 outlines possible steps. The observation that epithelial cells are not affected by purified $\mathrm{PVL}^{29}$ suggests that it is not directly responsible for tissue necrosis. Thus, PVL likely indirectly mediates tissue necrosis and sepsis by either the release of cytotoxic lysosomal granule contents from lysed PMNs or by an inflammatory cascade set in motion by PMN lysis or apoptosis. In support of this, PVL-mediated lysis induces the release of reactive oxygen species and a variety of inflammatory mediators from granulocytes. ${ }^{11}$

\section{Studies of PVL Pathogenesis in Animal Models}

One challenge has been to identify an animal model that closely mimics the severe disease manifested in humans. Several studies have demonstrated a role for PVL in dermonecrosis. ${ }^{23,26,27}$ In the only study to investigate invasive CA-MRSA infection, pvl-positive, CA-MRSA and MSSA clinical isolates were significantly more virulent by indices of morbidity, mortality, dissemination to various organs and histopathology compared with HA-MRSA lacking $p v l^{30}$ The fact that the CA strains were $p v l$-positive but from different genetic backgrounds suggests that PVL might be the common factor responsible for the observed increased virulence. Pvl-positive strains also were associated with a greater lysis of and survival in human PMNs. ${ }^{30}$ Although both HA- and CA-MRSA responded to PMNs by increasing transcription of the genes encoding gamma hemolysin, ${ }^{30}$ a bicomponent leukocidin structurally related to PVL, ${ }^{11}$ an assessment of the level of $p v l$ expression in the presence of PMNs was notably missing. Thus, it is unclear whether pvl expression is correlated with PMN lysis or virulence. In a separate study,

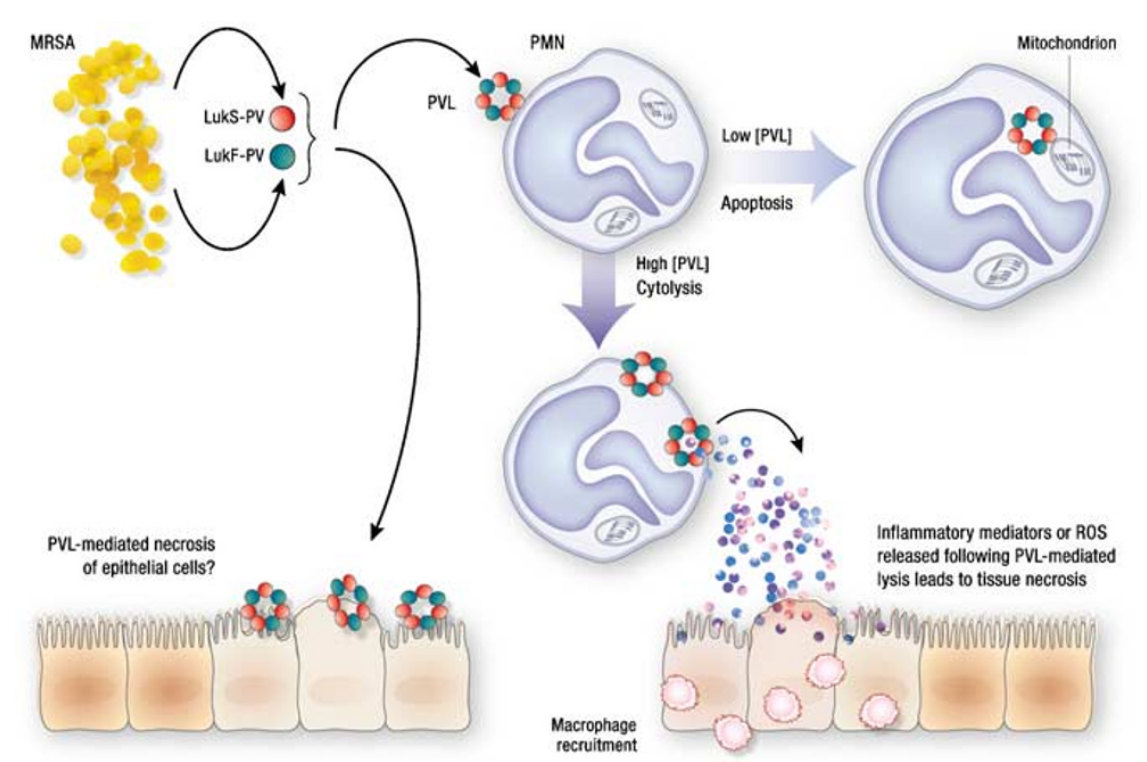

Figure 2 Model for how PVL might mediate tissue necrosis. The two components of PVL, LukS-PV and LukF-PV are secreted from $S$. aureus before they assemble into a pore-forming heptamer on PMN membranes. High PVL concentrations cause PMN lysis whereas low concentrations mediate a novel pathway of PMN apoptosis by directly binding to mitochondrial membranes. ${ }^{28}$ Tissue necrosis could result from release of reactive oxygen species (ROS) from lysed PMNs. Alternately, release of granule contents from lysed PMNs could set in motion an inflammatory response, eventually resulting in tissue necrosis. It is unlikely that PVL has a direct necrotic effect on epithelial cells. 


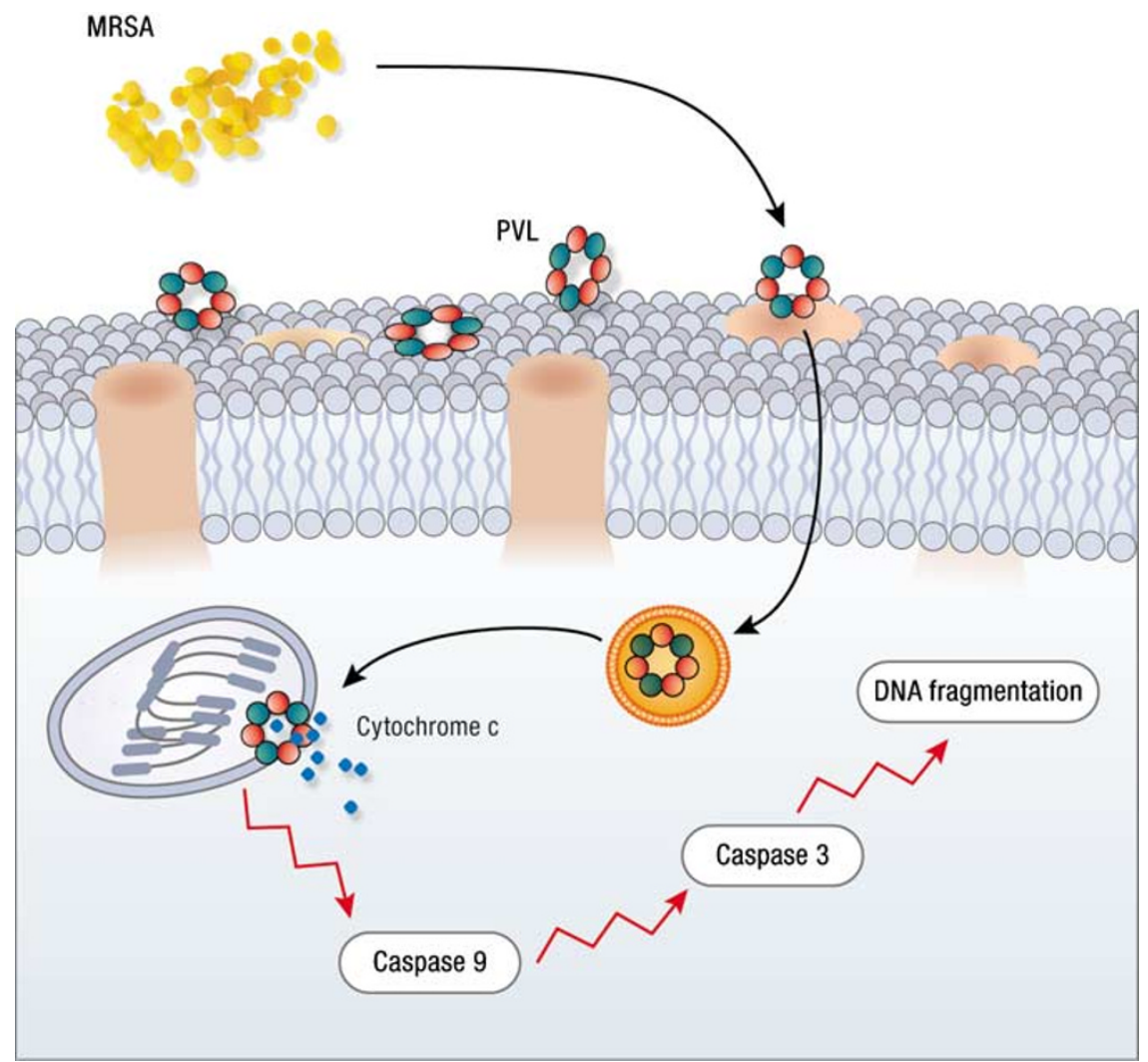

Figure 3 Unique mechanism of PVL-induced PMN apoptosis. Low PVL concentrations can induce apoptosis via a novel pathway that presumably involves PVL-mediated pore formation on the mitochondrial membrane leading to release of cytochrome $c$ and induction of caspases 9 and $3 .^{28}$

Said-Salim et $a l^{31}$ failed to identify a difference between pvl-positive and pvl-negative clinical isolates in their ability to lyse human PMNs; however, the pvl-positive isolates tested appeared to express $p v l$ at low levels. Strains expressing higher levels might have demonstrated a greater difference compared with pvl-negative strains. Nevertheless, even low PVL concentrations can lead to destruction of PMNs by mediating apoptosis. ${ }^{28}$ To determine the relative roles of apoptosis vs PMN lysis in pathogenesis, it will be important to determine the amount of PVL produced in situ during infection.

\section{Is a PVL Vaccine Justified?}

There is currently no vaccine licensed for $S$. aureus. Owing to the increasing spread of highly virulent pvl-positive strains it is perhaps time to consider whether a vaccine against PVL could eliminate pvl-positive invasive disease. Human intravenous polyclonal immunoglobulin (IVIG) therapy has been included in successful therapy regimens in the treatment of MRSA/MSSA severe sepsis at our institution $^{5}$ as well as elsewhere. Although the rationale for IVIG use cannot be directly attributed to the presence of anti-PVL antibodies, IVIG has been shown to bind to recombinant LukS-PV and
LukF-PV components. ${ }^{32}$ Furthermore, a commercial IVIG preparation neutralized pore formation in PMNs and the cytopathic effect of recombinant PVL. Immunization with purified PVL components conferred some protection against PVL-induced dermonecrosis. ${ }^{23,26}$ These data suggest that further studies are warranted to determine the usefulness of anti-PVL antibody in therapy or perhaps a PVLbased vaccine in disease prevention.

In conclusion, virulent, pvl-positive, CA-MRSA isolates have been emerging around the world in different $S$. aureus genetic lineages and are beginning to encroach on healthcare environments where they could become endemic. Multiple resistance to non- $\beta$-lactams has been reported in a few CA-MRSA isolates ${ }^{13,18}$ and this could become more common. Even resistance against mupirocin, often used for decolonization of patients with MRSA, has been reported among CA-MRSA isolates. ${ }^{18}$ The strong epidemiologic link between PVL and CA-MRSA disease leaves little doubt that PVL must play an important role in the pathogenesis and course of disease; however, the precise role of PVL in pathogenesis remains to be elucidated. Recent evidence suggests that in addition to the destruction of PMNs and/or monocytes and induction of inflammation, PVL might also enhance virulence indirectly by inducing expression of other virulence 
8

factors. Further understanding will be gained by continued monitoring of the molecular epidemiology and by the use of isogenic pvl-positive and pvl-negative bacterial strains in animal models of infection.

\section{Acknowledgements}

We apologize for omission of any pertinent references due to limitations imposed by the journal guidelines. RSD and SB-V are the recipients of R01 AI40481-01A1 from NIAID, RO1 CCR523379 and R01 C1000373-01 from the Centers for Disease Control and Prevention and support from the Grant HealthCare Foundation.

\section{References}

1 Lowy FD. Staphylococcus aureus infections. N Engl J Med 1998;339:520-532.

2 Carleton HA, Diep BA, Charlebois ED, et al. Community-adapted methicillin-resistant Staphylococcus aureus (MRSA): population dynamics of an expanding community reservoir of MRSA. J Infect Dis 2004;190:1730-1738.

3 Herold BC, Immergluck LC, Maranan M, et al. Community-acquired methicillin-resistant Staphylococcus aureus in children with no predisposing risk. JAMA 1997;279:593-598.

4 Vandenesch F, Naimi T, Enright MC, et al. Communityacquired methicillin-resistant Staphylococcus aureus carrying Panton-Valentine leukocidin genes: worldwide emergence. Emerg Infect Dis 2003;9:978-984.

5 Adem PV, Montgomery CP, Husain AN, et al. Staphylococcus aureus sepsis and the Waterhouse-Friderichsen syndrome in children. New Engl J Med 2005;353: 1245-1251.

6 Mongkolrattanothai K, Boyle S, Kahana MD, et al. Severe Staphylococcus aureus infections caused by clonally related community-acquired methicillin-susceptible and methicillin-resistant isolates. Clin Infect Dis 2003;37:1050-1058.

7 Moran GJ, Krishnadasan A, Gorwitz RJ, et al. Methicillin-resistant $S$. aureus infections among patients in the emergency department. N Engl J Med 2006;355: 666-674.

8 Miller LG, Perdreau-Remington F, Rieg G, et al. Necrotizing fasciitis caused by community-associated methicillin-resistant Staphylococcus aureus in Los Angeles. N Engl J Med 2005;352:1445-1453.

9 Gillet Y, Issartel B, Vanhems P, Godail-Gamot, et al. Association between Staphylococcus aureus strains carrying gene for Panton-Valentine leukocidin and highly lethal necrotising pneumonia in young immunocompetent patients. Lancet 2002;359:753-759.

10 Lina G, Piemont $\mathrm{Y}$, et al. Involvement of PantonValentine leukocidin-producing Staphylococcus aureus in primary skin infections and pneumonia. Clin Infect Dis 1999;29:1128-1132.

11 Kaneko J, Kamio Y. Bacterial two-component and hetero-heptameric pore-forming cytolytic toxins: structures, pore-forming mechanism, and organization of the genes. Biosci Biotechnol Biochem 2004;68: 981-1003.

12 Diep BA, Carleton HA, Chang RF, et al. Roles of 34 virulence genes in the evolution of hospital- and community-associated strains of methicillin-resistant Staphylococcus aureus. J Infect Dis 2006;193: 1495-1503.

13 Boyle-Vavra S, Ereshefsky B, Wang CC, et al. Successful multiresistant community-associated methicillinresistant Staphylococcus aureus lineage from Taipei, Taiwan, that carries either the novel staphylococcal chromosome cassette mec (SCCmec) type $V_{\mathrm{T}}$ or SCCmec type IV. J Clin Microbiol 2005;43:4719-4730.

14 Ito $\mathrm{T}, \mathrm{Ma} \mathrm{XX}$, Takeuchi $\mathrm{F}$, et al. Novel type V staphylococcal cassette chromosome mec driven by a novel cassette chromosome recombinase, ccrC. Antimicrob Agents Chemother 2004;48:2637-2651.

15 Ito T, Katayama Y, Asada K, et al. Structural comparison of three types of staphylococcal cassette chromosome mec in the chromosome of methicillin-resistant Staphylococcus aureus. Antimicrob Agents Chemother 2001;45:1323-1336.

16 Ma XX, Ito T, Tiensasitorn $\mathrm{C}$, et al. A novel type of staphylococcal cassette chromosome mec (SCCmec) identified in community-acquired methicillin-resistant Staphylococcus aureus strains. Antimicrob Agents Chemother 2002;46:1147-1152.

17 Daum RS, Ito T, Hiramatsu K, et al. A novel methicillin-resistance cassette in community-acquired methicillin-resistant Staphylococcus aureus isolates of diverse genetic backgrounds. J Infect Dis 2002;186: 1344-1347.

18 Diep BA, Gill SR, Chang RF, et al. Complete genome sequence of USA300, an epidemic clone of community-acquired methicillin-resistant Staphylococcus aureus. Lancet 2006;367:731-739.

19 Wisplinghoff H, Rosato AE, Enright MC, et al. Related clones containing SCCmec type IV predominate among clinically significant Staphylococcus epidermidis isolates. Antimicrob Agents Chemother 2003;47: 3574-3579.

20 Robinson DA, Enright MC. Multilocus sequence typing and the evolution of methicillin-resistant Staphylococcus aureus. Clin Microbiol Infect 2004;10:92-97.

21 McDougal LK, Steward CD, Killgore GE, et al. Pulsedfield gel electrophoresis typing of oxacillin-resistant Staphylococcus aureus isolates from the United States: establishing a national database. J Clin Microbiol 2003; 41:5113-5120.

22 Enright MC, Robinson DA, Randle G, et al. The evolutionary history of methicillin-resistant Staphylococcus aureus (MRSA). Proc Natl Acad Sci 2002;99: 7687-7692.

23 Ward PD, Turner WH. Identification of staphylococcal Panton-Valentine leukocidin as a potent dermonecrotic toxin. Infect Immun 1980;28:393-397.

24 Bogaert D, van Belkum A, Sluijter M, et al. Colonisation by Streptococcus pneumoniae and Staphylococcus aureus in healthy children. Lancet 2004;363: 1871-1872.

25 Maree C, Daum R, Boyle-Vavra S, et al. Rapid temporal increase in community-acquired methicillin-resistant Staphylococcus aureus strains causing nosocomial infections. In: Interscience Conference on Antimicrobial Agents and Chemotherapy. Washington, DC, 2006.

26 Cribier B, Prevost G, Couppie P, et al. Staphylococcus aureus leukocidin: a new virulence factor in cutaneous 
infections? An epidemiological and experimental study. Dermatology 1992;185:175-180.

27 Prevost G, Cribier B, Couppie P, et al. PantonValentine leucocidin and gamma-hemolysin from Staphylococcus aureus ATCC 49775 are encoded by distinct genetic loci and have different biological activities. Infect Immun 1995;63:4121-4129.

28 Genestier AL, Michallet MC, Prevost G, et al. Staphylococcus aureus Panton-Valentine leukocidin directly targets mitochondria and induces Bax-independent apoptosis of human neutrophils. J Clin Invest 2005; 115:3117-3127.

29 de Bentzmann S, Tristan A, Etienne J, et al. Staphylococcus aureus isolates associated with necrotizing pneumonia bind to basement membrane type I and IV collagens and laminin. J Infect Dis 2004;190: 1506-1515.

30 Voyich JM, Braughton KR, Sturdevant DE, et al. Insights into mechanisms used by Staphylococcus aureus to avoid destruction by human neutrophils. J Immunol 2005;175:3907-3919.

31 Said-Salim B, Mathema B, Braughton $\mathrm{K}$, et al. Differential distribution and expression of PantonValentine leucocidin among community-acquired methicillin-resistant Staphylococcus aureus strains. J Clin Microbiol 2005;43:3373-3379.

32 Gauduchon V, Cozon G, Vandenesch F, et al. Neutralization of Staphylococcus aureus Panton-Valentine leukocidin by intravenous immunoglobulin in vitro. J Infect Dis 2004;189:346-353. 